\title{
On the linear theory of micropolar plates
}

\author{
Holm Altenbach ${ }^{1, *}$ and Victor A. Eremeyev ${ }^{2, * *}$ \\ ${ }^{1}$ Lehrstuhl für Technische Mechanik, Zentrum für Ingenieurwissenschaften, Martin-Luther-Universität Halle-Wittenberg, \\ 06099 Halle (Saale), Germany \\ ${ }^{2}$ South Scientific Center of RASci \& South Federal University, Milchakova St. 8a, 344090 Rostov on Don, Russia
}

Key words Cosserat continuum, micropolar continuum, micropolar plates, effective stiffness.

Dedicated to Professor Vladimir A. Palmov on the occasion of his $75^{\text {th }}$ birthday.

We discuss the general linear six-parametric theory of plates based on the direct approach. We consider the plate as a deformable surface. Each material point of the surface can be regarded as an infinitesimal small rigid body with six degrees of freedom. The kinematics of the plate is described by using the vector of translation and the vector of rotation as the independent variables. The relations between the equilibrium conditions of a three-dimensional micropolar plate-like body and the two-dimensional equilibrium equations of the deformable surface are established. Using the three-dimensional constitutive equations of a micropolar material we discuss the determination of the effective stiffness tensors appearing in the two-dimensional constitutive equations.

\section{Introduction}

Mechanics of generalized continuum which differs from the classical (or Cauchy type) continuum mechanics is an old branch of mechanics with origins in the mid of the 19th century. On the other hand, it is in current and emerging interest of mechanicians, physicians, materials scientists as well as engineers, since the limits and possibilities of such theories are not fully known. One of such generalized continuum models is the micropolar continuum. In the micropolar continuum (also called the Cosserat continuum in some publications), each material particle has six degrees of freedom. From the physical point of view, every material point (or particle) of a micropolar medium is phenomenologically equivalent to a rigid body. Hence, rotations of the particles of the continuum are taken into account. The main ideas leading to the micropolar continuum and other generalized media were discussed at the end of 20 th century by Kelvin, Helmholtz, Duhem, Voigt, and Cosserat among others. The governing equations of the three-dimensional micropolar continuum as well as its onedimensional and two-dimensional analogues were presented in detail by E. and F. Cosserat in 1909 [13]. Since the mid of 20th century the interest to Cosserat's ideas is growing again and many publications devoted to the Cosserat continuum have appeared.

Since the paper of Ericksen \& Truesdell [23] the Cosserat model has found applications in construction of various generalized models for beams, plates, and shells. Within the framework of the direct approach applied in [23], the shell is modeled as a deformable surface at each point of which the set of deformable directors is attached. Hence, in general the deformation of a shell is described by the position vector $r$ and $p$ directors $d_{i}, i=1, \ldots p$. This approach was developed in the original papers by Ericksen [20-22], Green \& Naghdi [30-34], Green et al. [35]. This variant of shell theory is also named the Cosserat shell theory or the theory of Cosserat surfaces. There exists some criticism concerning the direct approach in general and especially the theory of Cosserat surfaces, see, for example, [69]. Let us mention the fundamental books by Naghdi [46], Rubin [64], and Antman [11] where the theory of Cosserat shells is presented. The theory of Cosserat shells contains as a special case the linear theory of Cosserat plates. This theory is mostly formulated with the help of introduction of one deformable director [30,31].

Independently Eringen has formulated a linear theory of micropolar plates in [24], see also the monograph [25]. The two-dimensional equations of this theory were deduced with the help of the independent integration over the thickness of the first and the second Euler laws of motion of the linear elastic micropolar continuum. The theories of the zeroth and the first order are presented applying a special linear approximation of the displacement and microrotation fields. Eringen's

* Corresponding author E-mail: holm.altenbach@iw.uni-halle.de, Phone: +49(0)345-5528430/1 Fax: +49(0)345-5527359

** E-mail: eremeyev.victor@gmail.com 
theory is based on eight unknowns: the averaged displacements, the averaged macrorotations of the cross-sections, and the averaged microrotations. This means that one has to introduce eight boundary conditions. The static boundary conditions in Eringen's plate cannot be presented as forces and moments at the boundaries like in the Kirchhoff type theories [70]. From the point of view of the direct approach Eringen's micropolar plate is a deformable surface with eight degrees of freedom.

The theories of plates and shells and the theories based on the reduction of the three-dimensional equations of the micropolar continuum are presented in various publications. In $[10,29,61,63]$ various averaging procedures in the thickness direction together with the approximation of the displacements and rotations or the force and moment stresses in the thickness direction are applied. As a result, one gets different numbers of unknowns and the number of two-dimensional equilibrium equations differs. For example, Reissner [63] presented a generalized linear theory of shells containing nine equilibrium equations. In addition, Reissner worked out the two-dimensional theory of a sandwich plate with a core having the properties of the Cosserat continuum [62]. The variants of the micropolar plate theory based on the asymptotic methods were developed in $[1,14,47,65,66]$. The $\Gamma$-convergence based approach to the theory of plates and shells was developed in $[48,49,51]$.

The direct approach in the theory of shells based on Cosserat's ideas was applied also in [72]. In contrast to [23], shells are regarded as deformable surfaces with material points at which three directors are prescribed. The directors have the following properties: they are orthogonal unit vectors. The deformations of the shell are presented by a position vector and a properly orthogonal tensor. This variant of shell theory based on the direct approach was developed and continued, for example, in $[15,18,19,67,68]$. It must be noted that this variant is very similar to the one presented within the general non-linear theory of shells and discussed in the monographs of Libai \& Simmonds [42] and Chróścielewski et al. [12], see also $[16,17,42,69]$. The two-dimensional equilibrium equations given in $[12,42]$ one gets by exact integration over the thickness of the equations of motion of a shell-like body. The deformation measures, which are the same as those introduced within the framework of the direct approach, can be defined as work-conjugate fields to the stress and the stress couple tensors.

In [8] the general theory with six degrees of freedom was transformed to theory of shells with five degrees of freedom (similar to the Reissner theory) introducing some constraints for the deformations. This variant of the theory was discussed in $[2-4,6,9,73]$. In [36] the method presented in [8] was applied to the three-dimensional case. It must be noted that the main problem in application of the direct approach is the definition of the constitutive equations. They should be formulated for the two-dimensional measures of stresses and measures of deformations. This means that some effective stiffness properties should be introduced. For anisotropic elastic plates the identification procedure for the effective stiffness properties is discussed in $[3,4,6,8,73]$, for the viscoelastic case in [2,5,7].

The aim of this paper is the discussion of a six-parameter linear theory of plates within the framework of the direct approach. The plate is regarded as a deformable surface with material points which have the same number of degrees of freedom as a rigid body. The deformations are described by two fields: the translation and the rotation fields. This theory will be named also the theory of micropolar plates, but it should be noted that the theory is different from Eringen's theory of micropolar plates as will be demonstrated later.

This paper can be regarded as a further development of the papers [53,54], which were published in the early 80th of the last century and which are not well-known. In those papers the direct approach was applied and some effective stiffness properties were determined. The present theory can be regarded as a special case of the six-parameter theory presented in $[12,19,42]$. But it should be noted that with the help of this special case one can better explain the interlink between the direct approach and the approaches which are based on the reduction of the three-dimensional theories. In addition, one can get an answer on the question where the two-dimensional and the three-dimensional equations are interlinked. It should be underlined that the presented plate model is more general in comparison with the Kirchhoff or Reissner plate models. The interaction between different parts of the two-dimensional Cosserat continuum is realized by forces and moments, distributed on the lines interlinking the parts. It should be noted that the vector of the distributed inner moment may have an arbitrary direction which is not the case for the Kirchhoff or Reissner plate theories. The advantage of the suggested theory in comparison with the more complex theories (for example, Eringen's theory of micropolar plates, Cosserat's surfaces or third-order theories) is that any interaction can be represented by forces and moments alone and not by higher-order stress resultants such as, for example, bi-moments or dipoles. The general order of the system of differential equations here is equal to twelve. In this case one has to formulate six boundary conditions. On the free boundary one has to prescribe distributed force and moment loadings. This means that the plate can carry a moment loading with arbitrary direction, which is distributed on the surface and along the boundary contour.

The paper is organized as follows. In Sect. 2 we present the governing equations of the linear micropolar plate theory formulated within the framework of the direct approach. We consider a micropolar plate as a deformable surface which the deformation is described by the fields of the translation vector $\boldsymbol{v}$ and the rotation vector $\boldsymbol{\theta}$. In other words, we consider a plate as a two-dimensional Cosserat continuum. The equilibrium equations are deduced and the linear constitutive equations are presented. Following [25], in Sect. 3 we recall the basic equations of the linear theory of micropolar elasticity. In Sects. 4 
and 5 we discuss the relation between the two-dimensional and three-dimensional equilibrium conditions as well as the reduction of the three-dimensional constitutive equations to the two-dimensional ones. For the homogeneous plate-like body we calculate the effective stiffness properties using special approximation of the translation and microrotation vectors of the micropolar plate-like body. In particular, we discuss the elastic stiffness corresponding to the drilling moment. An example of calculations of the elastic stiffness properties for a plate made of two porous materials is given in Sect. 6 .

\section{Micropolar plates equations based on the direct approach}

In this section we present the basic equations of the theory of micropolar plates based on the so-called direct approach. Within the framework of the direct approach we consider a micropolar plate as a two-dimensional Cosserat continuum. In other words, we consider a micropolar plate as a deformable plane surface, see Fig. 1. Each material point of the surface is an infinitesimal rigid body with 6 degrees of freedom ( 3 translations and 3 rotations). In addition, the theory presented here is limited by small displacements and rotations and the quadratic strain energy function. Hence, the deformations of the micropolar plate are described by the translation vector $\boldsymbol{v}$ and the rotation vector $\boldsymbol{\theta}$ which are defined on the surface $\mathcal{M}$.

The basics of the non-linear theory of micropolar shells are presented in $[8,9,15,18,19,72,73]$. Let us note that the governing equations of the micropolar shells and plates coincide with the relations of the general 6-parametric nonlinear shell theory presented in $[12,16,17,41,42]$. The linear variant of this theory is given in [12].

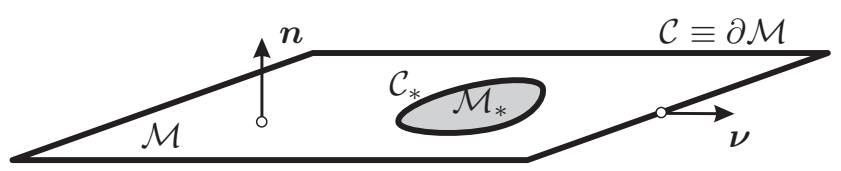

Fig. 1 Deformable plane surface.

Within the framework of the direct approach the balance of momentum and the balance of moment of momentum are formulated as follows

$$
\mathcal{F}_{s}^{*} \equiv \int_{\mathcal{M}_{*}} \boldsymbol{q} \mathrm{d} A+\int_{\mathcal{C}_{*}} \boldsymbol{t}_{s} d s=\mathbf{0}, \quad \mathcal{M}_{s}^{*} \equiv \int_{\mathcal{M}_{*}}(\boldsymbol{x} \times \boldsymbol{q}+\boldsymbol{c}) \mathrm{d} A+\int_{\mathcal{C}_{*}}\left(\boldsymbol{x} \times \boldsymbol{t}_{s}+\boldsymbol{m}_{s}\right) \mathrm{d} s=\mathbf{0},
$$

where $\boldsymbol{q}$ and $\boldsymbol{c}$ are the surface loads (forces and moments), $\boldsymbol{x}$ is the position vector of the plane surface $\mathcal{M}, \times$ is the cross product, $\boldsymbol{t}_{s}$ and $\boldsymbol{m}_{s}$ are the surface analogues of the stress vector and the couple stress vector, respectively. From Eqs. (1) we obtain the local form of the balances of momentum and moment of momentum as well as the static boundary conditions. Using the surface analogues of the Cauchy theorem we introduce the force tensor $\mathbf{T}$ and the moment tensor $\mathbf{M}$ by the relations

$$
\nu \cdot \mathbf{T}=t_{s}, \quad \nu \cdot \mathrm{M}=\boldsymbol{m}_{s},
$$

where $\boldsymbol{\nu}$ is the unit vector externally normal to $\mathcal{C}(\boldsymbol{\nu} \cdot \boldsymbol{n}=0)$, and $\cdot$ is the dot product. Then the local equilibrium equations are

$$
\nabla_{s} \cdot \mathbf{T}+\boldsymbol{q}=\mathbf{0}, \quad \nabla_{s} \cdot \mathbf{M}+\mathbf{T}_{\times}+\boldsymbol{c}=\mathbf{0},
$$

where $\mathbf{T}_{\times}$denotes the vector invariant of the second-rank tensor $\mathbf{T}$ defined by the relation $\mathbf{T}_{\times} \equiv\left(T_{m n} \boldsymbol{i}_{m} \otimes \boldsymbol{i}_{n}\right)_{\times}=$ $T_{m n} \boldsymbol{i}_{m} \times \boldsymbol{i}_{n}{ }^{1}, \nabla_{s}$ is the surface (plane) nabla (Hamilton) operator, $\nabla_{s}=\boldsymbol{i}_{\alpha} \frac{\partial}{\partial x_{\alpha}}, \nabla_{s} \boldsymbol{u}=\boldsymbol{i}_{\alpha} \otimes \boldsymbol{u}_{, \alpha}, \boldsymbol{i}_{1}, \boldsymbol{i}_{2}, \boldsymbol{i}_{3}$ are the Cartesian base vectors, $(\ldots)_{, \alpha}$ is the partial derivative with respect to the Cartesian coordinate $x_{\alpha}, \alpha=1,2$. The tensors $\mathbf{T}$ and $\mathbf{M}$ have the properties

$$
n \cdot \mathrm{T}=\mathbf{0}, \quad n \cdot \mathrm{M}=\mathbf{0} .
$$

Hence, $\mathbf{T}$ and $\mathbf{M}$ take the form

$$
\mathbf{T}=T_{\alpha \beta} \boldsymbol{i}_{\alpha} \otimes \boldsymbol{i}_{\beta}+T_{\alpha 3} \boldsymbol{i}_{\alpha} \otimes \boldsymbol{n}, \quad \mathbf{M}=M_{\alpha \beta} \boldsymbol{i}_{\alpha} \otimes \boldsymbol{i}_{\beta}+M_{\alpha 3} \boldsymbol{i}_{\alpha} \otimes \boldsymbol{n} \quad(\alpha, \beta=1,2) .
$$

Here we assume that $\boldsymbol{i}_{3}=\boldsymbol{n}$. Let us note that in the literature on shell they use another coordinate representation for $\mathbf{M}$ :

$$
\mathbf{M}=\boldsymbol{i}_{\alpha} \otimes \boldsymbol{m}_{\alpha}=i_{\alpha} \otimes\left[\boldsymbol{n} \times M_{\alpha \beta} \boldsymbol{i}_{\beta}+M_{\alpha 3} \boldsymbol{n}\right] .
$$

Here we will use (4) $)_{2}$ for the sake of simplicity.

\footnotetext{
1 This operation was introduced originally by J. W. Gibbs, see [71]
} 
In Cartesian coordinates Eqs. (2) can be transformed to

$$
\begin{aligned}
& T_{11,1}+T_{21,2}+q_{1}=0, \quad M_{11,1}+M_{21,2}+T_{23}+c_{1}=0, \\
& T_{12,1}+T_{22,2}+q_{2}=0, \quad M_{12,1}+M_{22,2}-T_{13}+c_{2}=0, \\
& T_{13,1}+T_{23,2}+q_{3}=0, \quad M_{13,1}+M_{23,2}+T_{12}-T_{21}+c_{3}=0,
\end{aligned}
$$

where $q_{m}=\boldsymbol{q} \cdot \boldsymbol{i}_{m}, c_{m}=\boldsymbol{c} \cdot \boldsymbol{i}_{m}, m=1,2,3$. It is evident that in this theory the action of the drilling moment $c_{3}$ is possible to take into account. For example, such possibility may be useful to describe the interaction of the plate and the rigid body or for description of the deformations of multifolded plates.

The static and kinematic boundary conditions take the form

$$
\boldsymbol{\nu} \cdot \mathbf{T}=\boldsymbol{t}_{s}^{*}, \quad \boldsymbol{\nu} \cdot \mathbf{M}=\boldsymbol{m}_{s}^{*} \quad \text { along } \mathcal{C}_{f}, \quad \boldsymbol{v}=\boldsymbol{v}^{0}, \quad \boldsymbol{\theta}=\boldsymbol{\theta}^{0} \quad \text { along } \mathcal{C}_{u} .
$$

Here $\boldsymbol{t}_{s}^{*}$ and $\boldsymbol{m}_{s}^{*}$ are external force and couple vectors acting along the boundary of plate $\mathcal{C}_{f}$, while $\boldsymbol{u}^{0}$ and $\boldsymbol{\theta}^{0}$ are given functions describing displacements and rotations of the plate boundary $\mathcal{C}_{u}$, respectively.

The linear strain measures are

$$
\boldsymbol{\epsilon}=\nabla_{s} \boldsymbol{v}+\mathbf{A} \times \boldsymbol{\theta}, \quad \boldsymbol{\kappa}=\nabla_{s} \boldsymbol{\theta},
$$

where $\mathbf{A} \equiv \mathbf{I}-\boldsymbol{n} \otimes \boldsymbol{n}$ is the first metric tensor (two-dimensional or plane unit tensor). Note that when the plate deformation corresponds to the rigid body motion, i.e. when $\boldsymbol{v}=\boldsymbol{a}+\boldsymbol{\omega} \times \boldsymbol{x}, \boldsymbol{\theta}=\boldsymbol{\omega}$ with the constant vectors $\boldsymbol{a}, \boldsymbol{\omega}$, then $\boldsymbol{\epsilon}=\boldsymbol{\kappa}=\mathbf{0}$.

Using the technique presented in $[12,42]$ one may show that the linear strain measures (7) are work-conjugate to the stress measures $\mathbf{T}$ and $\mathbf{M}$. Indeed, let us introduce two arbitrary smooth vector fields $\boldsymbol{w}$ and $\boldsymbol{\omega}$. By dot multiplying the equilibrium equations (2) and the static boundary conditions (6) on $\boldsymbol{w}$ and $\boldsymbol{\omega}$, we obtain the integral identity

$$
\int_{\mathcal{M}}\left[\left(\nabla_{s} \cdot \mathbf{T}+\boldsymbol{q}\right) \cdot \boldsymbol{w}+\left(\nabla_{s} \cdot \mathbf{M}+\mathbf{T}_{\times}+\boldsymbol{c}\right) \cdot \boldsymbol{\omega}\right] \mathrm{d} A+\int_{\mathcal{C}_{f}}\left[\left(\boldsymbol{\nu} \cdot \mathbf{T}-\boldsymbol{t}_{s}^{*}\right) \cdot \boldsymbol{w}+\left(\boldsymbol{\nu} \cdot \mathbf{M}-\boldsymbol{m}_{s}^{*}\right) \cdot \boldsymbol{\omega}\right] \mathrm{d} s=0 .
$$

Using the identity $\mathbf{T}_{\times} \cdot \boldsymbol{\omega}=-\mathbf{T} \cdot(\mathbf{A} \times \boldsymbol{\omega})^{T}$, where $(\ldots)^{T}$ denotes the transpose, and the Gauss-Ostrogradski theorem we transform Eq. (8) to the form

$$
\begin{aligned}
\int_{\mathcal{M}} & {\left[\mathbf{T} \cdots\left(\nabla_{s} \boldsymbol{w}+\mathbf{A} \times \boldsymbol{\omega}\right)^{T}+\mathbf{M} \cdots\left(\nabla_{s} \boldsymbol{\omega}\right)^{T}\right] \mathrm{d} A } \\
& =\int_{\mathcal{M}}(\boldsymbol{q} \cdot \boldsymbol{w}+\boldsymbol{c} \cdot \boldsymbol{\omega}) \mathrm{d} A+\int_{\mathcal{C}_{f}}\left(\boldsymbol{t}_{s}^{*} \cdot \boldsymbol{w}+\boldsymbol{m}_{s}^{*} \cdot \boldsymbol{\omega}\right) \mathrm{d} s+\int_{\mathcal{C}_{u}}(\boldsymbol{\nu} \cdot \mathbf{T} \cdot \boldsymbol{w}+\boldsymbol{\nu} \cdot \mathbf{M} \cdot \boldsymbol{\omega}) \mathrm{d} s .
\end{aligned}
$$

The vector field $\boldsymbol{w}$, in particular, can be interpreted as the kinematically admissible virtual translation $\boldsymbol{w} \equiv \delta \boldsymbol{v}$ and the vector field $\boldsymbol{\omega}$ as the kinematically admissible virtual rotation $\boldsymbol{\omega} \equiv \delta \boldsymbol{\theta}$, such that $\boldsymbol{w}=\boldsymbol{\omega}=\mathbf{0}$ on $\mathcal{C}_{u}$, where $\delta$ is the symbol of variation. If one assumes that $\boldsymbol{w} \equiv \delta \boldsymbol{v}$ and $\boldsymbol{\omega} \equiv \delta \boldsymbol{\theta}$ then we obtain $\nabla_{s} \boldsymbol{w}+\mathbf{A} \times \boldsymbol{\omega}=\delta \boldsymbol{\epsilon}$ and $\nabla_{s} \boldsymbol{\omega}=\delta \boldsymbol{\kappa}$. Then the last line integral in (9) identically vanishes, two integrals in the second row of (9) describe the external virtual work, while the first volume integral in (9) describes the internal virtual work performed by the stress measures on the work-conjugate virtual strain measures, that means Eq. (9) represents the principle of virtual work

$$
\int_{\mathcal{M}}\left(\mathbf{T} \cdot \delta \boldsymbol{\epsilon}^{T}+\mathbf{M} \cdot \delta \boldsymbol{\kappa}^{T}\right) \mathrm{d} A=\int_{\mathcal{M}}(\boldsymbol{q} \cdot \boldsymbol{w}+\boldsymbol{c} \cdot \boldsymbol{\omega}) \mathrm{d} A+\int_{\mathcal{C}_{f}}\left(\boldsymbol{t}_{s}^{*} \cdot \boldsymbol{w}+\boldsymbol{m}_{s}^{*} \cdot \boldsymbol{\omega}\right) \mathrm{d} s .
$$

The internal virtual work density in (10) is given by the expression $\sigma=\mathbf{T} \cdot \delta \boldsymbol{\epsilon}^{T}+\mathbf{M} \cdot \delta \boldsymbol{\kappa}^{T}$. Hence, the linear strain measures $\epsilon$ and $\boldsymbol{\kappa}$ introduced in Sect. 2 are work-conjugate to the surface stress tensor $\mathbf{T}$ and the surface couple stress tensor $\mathbf{M}$, respectively. The introduction of the strain measures in the three-dimensional micropolar continuum as work-conjugate to the stress and couple stress tensors is discussed in [58]. 
For the isotropic plate the surface strain energy density is given by the relation $[17,19]$

$$
\begin{aligned}
2 W= & \alpha_{1} \operatorname{tr}^{2} \boldsymbol{\epsilon}_{\|}+\alpha_{2} \operatorname{tr} \boldsymbol{\epsilon}_{\|}^{2}+\alpha_{3} \operatorname{tr}\left(\boldsymbol{\epsilon}_{\|} \cdot \boldsymbol{\epsilon}_{\|}^{T}\right)+\alpha_{4} \boldsymbol{n} \cdot \boldsymbol{\epsilon}^{T} \cdot \boldsymbol{\epsilon} \cdot \boldsymbol{n} \\
& +\beta_{1} \operatorname{tr}^{2} \boldsymbol{\kappa}_{\|}+\beta_{2} \operatorname{tr} \boldsymbol{\kappa}_{\|}^{2}+\beta_{3} \operatorname{tr}\left(\boldsymbol{\kappa}_{\|} \cdot \boldsymbol{\kappa}_{\|}^{T}\right)+\beta_{4} \boldsymbol{n} \cdot \boldsymbol{\kappa}^{T} \cdot \boldsymbol{\kappa} \cdot \boldsymbol{n} .
\end{aligned}
$$

Here $\boldsymbol{\epsilon}_{\|}=\boldsymbol{\epsilon} \cdot \mathbf{A}, \boldsymbol{\kappa}_{\|}=\boldsymbol{\kappa} \cdot \mathbf{A}$, and $\alpha_{i}, \beta_{i}$ are the elastic constants, $i=1,2,3,4$. The surface stress and couple stress tensors take the form

$$
\begin{aligned}
& \mathbf{T} \equiv \frac{\partial W}{\partial \boldsymbol{\epsilon}}=\alpha_{1} \mathbf{A} \operatorname{tr} \boldsymbol{\epsilon}_{\|}+\alpha_{2} \boldsymbol{\epsilon}_{\|}^{T}+\alpha_{3} \boldsymbol{\epsilon}_{\|}+\alpha_{4} \boldsymbol{\epsilon} \cdot \boldsymbol{n} \otimes \boldsymbol{n}, \\
& \mathbf{M} \equiv \frac{\partial W}{\partial \boldsymbol{\kappa}}=\beta_{1} \mathbf{A} \operatorname{tr} \boldsymbol{\kappa}_{\|}+\beta_{2} \boldsymbol{\kappa}_{\|}^{T}+\beta_{3} \boldsymbol{\kappa}_{\|}+\beta_{4} \boldsymbol{\kappa} \cdot \boldsymbol{n} \otimes \boldsymbol{n} .
\end{aligned}
$$

For the homogeneous isotropic micropolar plates one can write the equilibrium equations (2) in terms of $\boldsymbol{v}$ and $\boldsymbol{\theta}$ taking into account the linear strain measures (7)) and the constitutive equations (12) and (13)

$$
\begin{aligned}
& \left(\alpha_{1}+\alpha_{2}\right) \nabla_{s} \nabla_{s} \cdot \boldsymbol{v}_{s}+\alpha_{3} \nabla_{s} \cdot \nabla_{s} \boldsymbol{v}_{s}+\alpha_{4} \nabla_{s} \cdot \nabla_{s}(\boldsymbol{v} \cdot \boldsymbol{n}) \boldsymbol{n}+\left(\alpha_{3}-\alpha_{2}\right) \nabla_{s}(\boldsymbol{\theta} \cdot \boldsymbol{n}) \times \boldsymbol{n} \\
& \quad+\alpha_{4}\left(\nabla_{s} \boldsymbol{\theta}_{s}\right)_{\times}+\boldsymbol{q}=\mathbf{0}, \\
& \left(\beta_{1}+\beta_{2}\right) \nabla_{s} \nabla_{s} \cdot \boldsymbol{\theta}_{s}+\beta_{3} \nabla_{s} \cdot \nabla_{s} \boldsymbol{\theta}_{s}+\beta_{4} \nabla_{s} \cdot \nabla_{s}(\boldsymbol{\theta} \cdot \boldsymbol{n}) \otimes \boldsymbol{n} \\
& \quad+\left(\alpha_{3}-\alpha_{2}\right) \nabla_{s} \times \boldsymbol{v}_{s}-2\left(\alpha_{3}-\alpha_{2}\right)(\boldsymbol{\theta} \cdot \boldsymbol{n}) \boldsymbol{n}+\alpha_{4}\left[\nabla_{s}(\boldsymbol{v} \cdot \boldsymbol{n}) \times \boldsymbol{n}-\boldsymbol{\theta}_{s}\right]+\boldsymbol{c}=\mathbf{0},
\end{aligned}
$$

where $\boldsymbol{v}_{s}=\boldsymbol{v} \cdot \mathbf{A}, \boldsymbol{\theta}_{s}=\boldsymbol{\theta} \cdot \mathbf{A}$.

The surface strain energy $W$ should be positive definite, from which follow the inequalities

$$
\begin{array}{llll}
2 \alpha_{1}+\alpha_{2}+\alpha_{3}>0, & \alpha_{2}+\alpha_{3}>0, & \alpha_{3}-\alpha_{2}>0, & \alpha_{4}>0, \\
2 \beta_{1}+\beta_{2}+\beta_{3}>0, & \beta_{2}+\beta_{3}>0, & \beta_{3}-\beta_{2}>0, & \beta_{4}>0 .
\end{array}
$$

Note that for an isotropic three-dimensional micropolar solid we have 6 elastic moduli while the micropolar plate Eq. (11) contains 8 elastic stiffness parameters. The increase of the number of parameters in the two-dimensional theory of plates in comparison with the three-dimensional theory can be related as the minimum to two items:

- Reduced symmetry of the constitutive equations. If we understand the constitutive equations for the plate as tensorial relations $\mathbf{T}=\mathbf{T}(\boldsymbol{\epsilon}, \boldsymbol{\kappa})$ and $\mathbf{M}=\mathbf{M}(\boldsymbol{\epsilon}, \boldsymbol{\kappa})$, these relations should be invariant with respect to some transformations which are elements of the symmetry group of the material. In the case of two-dimensional equations (plates, shells) a smaller number of elements belongs to the symmetry group than in the three-dimensional case. For example, in the three-dimensional theory of elasticity the constitutive equations for the isotropic material are invariant with respect to the rotations about an arbitrary axis, while for the isotropic shells the symmetry group contains only the rotations about the normal axis, see [17]. This means the symmetry properties for plates and shells are not so restrictive in comparison with the three-dimensional ones.

- Reduction of three-dimensional equations to two-dimensional. For the Kirchhoff theory, if this theory is considered within the direct approach, one obtains three independent stiffness parameters. They are determined by the following three parameters: the Young modulus of a bulk material $E$, the Poisson ratio $\nu$, and the plate thickness $h$. In the Reissner theory an additional property is the shear correction factor $k$, see $[37,70]$, which can be regarded as an independent property. So Reissner theory is related to four stiffness parameters in comparison with the theory of elasticity, where the two Lamè coefficients are enough. From this we can make the conclusion that in any plate or shell theory one can obtain an increase of parameters even in the Kirchhoff or Reissner theories.

Thus, one needs to identify these elastic moduli. The identification is not a trivial procedure even in the theory of elastic plates with less degrees of freedom. Therefore, one may

- determine $\alpha_{i}, \beta_{i}$ directly using the experimental data on thin-walled specimens;

- express $\alpha_{i}, \beta_{i}$ via the elastic moduli of the bulk material and the plate thickness.

Both ways have some advantages and disadvantages.

In [12] the following relations for the elastic moduli appearing in (11), (12), and (13) were used

$$
\alpha_{1}=C \nu, \quad \alpha_{2}=0, \quad \alpha_{3}=C(1-\nu), \quad \alpha_{4}=\alpha_{s} C(1-\nu),
$$




$$
\begin{array}{r}
\beta_{1}=D \nu, \quad \beta_{2}=0, \quad \beta_{3}=D(1-\nu), \quad \beta_{4}=\alpha_{t} D(1-\nu), \\
C=\frac{E h}{1-\nu^{2}}, \quad D=\frac{E h^{3}}{12\left(1-\nu^{2}\right)},
\end{array}
$$

where $\alpha_{s}$ and $\alpha_{t}$ are dimensionless coefficients. Note that in [12] it was assumed the linear isotropic behaviour of the bulk material considering the Hooke law. $\alpha_{s}$ is similar to the shear correction factor introduced in the plate theory by Reissner [59] $\left(\alpha_{s}=5 / 6\right)$ and by Mindlin [43] $\left(\alpha_{s}=\pi^{2} / 12\right)$, see also [37]. $\alpha_{t}$ plays the same role for the moment stresses. The value $\alpha_{t}=0.7$ was proposed in [56,57]. For several problems considered in [12] numerically the influence of $\alpha_{t}$ on the solution was analyzed. It was shown that for homogeneous shells with some boundary conditions this influence is insignificant if $\alpha_{t}<1$.

\section{Basic equations of the three-dimensional micropolar elasticity}

Following [25], in this section we recall the governing equations of the linear micropolar elasticity. Let the micropolar body occupies the domain $V \in \mathbb{R}^{3}$. The equilibrium conditions of any part of a micropolar body occupying the volume $V_{*} \subset V$ consist of the following relations

$$
\mathcal{F}^{*} \equiv \int_{V_{*}} \rho \boldsymbol{f} \mathrm{d} V+\int_{S_{*}} \boldsymbol{t} \mathrm{d} A=\mathbf{0}, \quad \mathcal{M}^{*} \equiv \int_{V_{*}} \rho(\boldsymbol{r} \times \boldsymbol{f}+\boldsymbol{\ell}) \mathrm{d} V+\int_{S_{*}}(\boldsymbol{r} \times \boldsymbol{t}+\boldsymbol{m}) \mathrm{d} A=\mathbf{0},
$$

where $\boldsymbol{f}$ and $\ell$ are the mass force and mass couple vectors, respectively, $\rho$ is the density, $\boldsymbol{r}$ is the position vector, $S_{*}=\partial V_{*}$, $\boldsymbol{t}$ and $\boldsymbol{m}$ are the stress and couple stress vectors, respectively, $\mathcal{F}^{*}$ and $\mathcal{M}^{*}$ are the total force and the total couple acting on $V_{*}$. Hence, for any part of the micropolar body Eq. (17) $)_{1}$ states that the total force is zero, while Eq. (17) $)_{2}$ states that the total moment is zero.

Using the Cauchy theorem we introduce the stress tensor $\sigma$ and the couple stress tensor $\mu$

$$
n \cdot \sigma=t, \quad n \cdot \mu=m,
$$

where $\boldsymbol{n}$ is the unit normal to $S_{*}$. From Eqs. (17) follow the local equilibrium equations and the static boundary conditions. The first ones are

$$
\nabla \cdot \boldsymbol{\sigma}+\rho \boldsymbol{f}=\mathbf{0}, \quad \nabla \cdot \boldsymbol{\mu}+\boldsymbol{\sigma}_{\times}+\rho \boldsymbol{\ell}=\mathbf{0},
$$

where $\nabla$ is the three-dimensional nabla operator, $\nabla=\nabla_{s}+\boldsymbol{i}_{3} \frac{\partial}{\partial z}$. Equation (19) 1 is the local form of the balance of momentum while Eq. $(19)_{2}$ is the balance of moment of momentum. In Cartesian coordinates these equilibrium equations are

$$
\sigma_{m n, m}+\rho f_{n}=0, \quad \mu_{m n, m}+\epsilon_{n p t} \sigma_{p t}+\rho \ell_{n}=0, \quad \boldsymbol{\sigma}=\sigma_{m n} \boldsymbol{i}_{m} \otimes \boldsymbol{i}_{n}, \quad \boldsymbol{\mu}=\mu_{m n} \boldsymbol{i}_{m} \otimes \boldsymbol{i}_{n} .
$$

The static boundary conditions have the following form

$$
\boldsymbol{n} \cdot \boldsymbol{\sigma}=\boldsymbol{t}^{0}, \quad \boldsymbol{n} \cdot \boldsymbol{\mu}=\boldsymbol{m}^{0} \quad \text { at } S_{f}
$$

Here $\boldsymbol{t}^{0}$ and $\boldsymbol{m}^{0}$ are the surface forces and the surface couples acting on the corresponding part of the surface $S_{f}$ of the micropolar body, $S=S_{u} \cup S_{f} \equiv \partial V$. The kinematic boundary conditions consist of the following relations

$$
\boldsymbol{u}=\boldsymbol{u}^{0}, \quad \boldsymbol{\vartheta}=\boldsymbol{\vartheta}^{0} \quad \text { at } S_{u}
$$

where $\boldsymbol{u}^{0}$ and $\boldsymbol{\vartheta}^{0}$ are given functions at $S_{u}$. Other types of the boundary conditions may also be formulated.

The small deformations of the micropolar media are usually described by using the vector of translation $\boldsymbol{u}$ and the vector of microrotation $\vartheta$. From the physical point of view, $\boldsymbol{u}$ describes a displacement of a particle of a micropolar body while $\vartheta$ corresponds to a particle rotation. The linear stain measures, i.e. the linear stretch tensor $\varepsilon$ and the linear wryness tensor $\mathfrak{x}$, are given by the relations

$$
\varepsilon=\nabla \boldsymbol{u}+\boldsymbol{\vartheta} \times \mathbf{I}, \quad æ=\nabla \boldsymbol{\vartheta},
$$

where $\mathbf{I}$ is the unit three-dimensional tensor. In [25] Eringen used $(\nabla \boldsymbol{\vartheta})^{T}$ as the linear wryness tensor. Here we use the definition $(22)_{2}$ for the consistency with the definition of $\varepsilon$. In Cartesian coordinates Eqs. (22) have the form

$$
\boldsymbol{\varepsilon}=\varepsilon_{m n} \boldsymbol{i}_{m} \otimes \boldsymbol{i}_{n}, \quad \varepsilon_{m n}=u_{n, m}+\epsilon_{n m k} \vartheta_{k}, \quad \mathfrak{x}=æ_{m n} \boldsymbol{i}_{m} \otimes \boldsymbol{i}_{n}, \quad æ_{m n}=\vartheta_{n, m} .
$$


For an isotropic solid the constitutive equations are

$$
\boldsymbol{\sigma}=\lambda \mathbf{I} \operatorname{tr} \boldsymbol{\varepsilon}+\mu \boldsymbol{\varepsilon}^{T}+(\mu+\kappa) \boldsymbol{\varepsilon}, \quad \boldsymbol{\mu}=\alpha \mathbf{I t r} \mathfrak{x}+\beta \mathfrak{x}^{T}+\gamma \mathfrak{e} .
$$

In Eqs. (23), $\lambda, \mu, \kappa, \alpha, \beta, \gamma$ are the elastic moduli which satisfy the inequalities [25,50]

$$
2 \mu+\kappa \geq 0, \quad \kappa \geq 0, \quad 3 \lambda+2 \mu+\kappa \geq 0, \quad \beta+\gamma \geq 0, \quad \gamma-\beta \geq 0, \quad 3 \alpha+\beta+\gamma \geq 0 .
$$

Within the framework of the micropolar continuum the experimental identification of the elastic moduli was discussed in $[27,28,38,40,45,55]$, see also the data in $[25,26]$ and the web site by P. Neff ${ }^{2}$. Substituting Eqs. (23) into Eqs. (19) one may derive the equilibrium equations in terms of the fields $\boldsymbol{u}$ and $\boldsymbol{\vartheta}$. For homogeneous micropolar bodies these equations are

$$
\begin{gathered}
(\lambda+\mu) \nabla \nabla \cdot \boldsymbol{u}+(\mu+\kappa) \nabla \cdot \nabla \boldsymbol{u}+\kappa \nabla \times \boldsymbol{\vartheta}+\rho \boldsymbol{f}=\mathbf{0}, \\
(\alpha+\beta) \nabla \nabla \cdot \boldsymbol{\vartheta}+\gamma \nabla \cdot \nabla \boldsymbol{\vartheta}+\kappa \nabla \times \boldsymbol{u}-2 \kappa \boldsymbol{\vartheta}+\rho \boldsymbol{\ell}=\mathbf{0} .
\end{gathered}
$$

If one assumes $\kappa=0$ then from Eq. (23) 1 it follows that $\boldsymbol{\sigma}=\boldsymbol{\sigma}^{T}$. In this case $\boldsymbol{\sigma}_{\times}=\mathbf{0}$ and the boundary-value problem (19), (20), and (21) splits into two independent BVPs formulated in terms of $\boldsymbol{u}$ and $\boldsymbol{\vartheta}$, respectively. In addition, if we assume that $\alpha=\beta=\gamma=0$ then $\boldsymbol{\mu} \equiv \mathbf{0}$ and with $\boldsymbol{\ell}=\boldsymbol{m}=\mathbf{0}$ the considered boundary-value problem reduces to the $\mathrm{BVP}$ of the classical linear elasticity. Thus, the equilibrium conditions of a three-dimensional micropolar medium contain the equilibrium conditions of the Cauchy continuum as a special case.

\section{Micropolar plate's equilibrium equations derived by the through-the-thickness in- tegration procedure}

In this section we show the relations between the three-dimensional and two-dimensional formulations of the Cosserat continuum presented above. Let us consider the plate-like body occupying the volume $V=\left\{(x, y, z) \in \mathbb{R}^{3}:(x, y) \in\right.$ $\left.\mathcal{M} \subset \mathbb{R}^{2}, z \in[-h / 2, h / 2]\right\}$, see Fig. 2. Here $h$ is the plate thickness. For the sake of simplicity we assume that $h=$ const. We denote the boundary of the plate-like body as $S=S^{\nu} \bigcup S^{+} \bigcup S^{-} \equiv \partial V$, where $S^{ \pm}=\{(x, y, z):(x, y) \in \mathcal{M}, z=$ $\pm h / 2$ and $S^{\nu}=\{(x, y, z):(x, y) \in \mathcal{C} \equiv \partial \mathcal{M}, z \in[-h / 2, h / 2]\}$, and assume the following boundary conditions at $S^{ \pm}$

$$
\boldsymbol{n}^{ \pm} \cdot \boldsymbol{\sigma}=\boldsymbol{t}^{ \pm}, \quad \boldsymbol{n}^{ \pm} \cdot \boldsymbol{\mu}=\boldsymbol{m}^{ \pm}
$$

where $\boldsymbol{t}^{ \pm}, \boldsymbol{m}^{ \pm}$are given functions, and $\boldsymbol{n}^{ \pm}= \pm \boldsymbol{i}_{3}$.

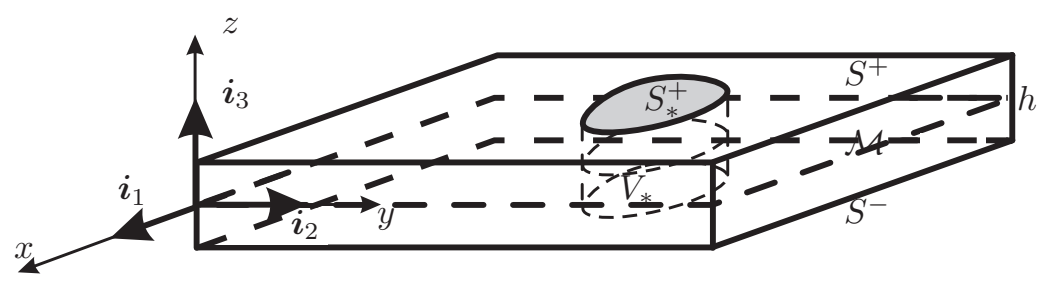

Fig. 2 Plate-like body.

Let us apply the integral equilibrium conditions (17) to the plate-like body with $V_{*}=\left\{(x, y, z):(x, y) \in \mathcal{M}_{*} \subset \mathcal{M}\right.$, $z \in[-h / 2, h / 2]\}$ and $S_{*} \equiv \partial V_{*}=S_{*}^{\nu} \bigcup S_{*}^{+} \bigcup S_{*}^{-}$, see Fig. 2. Note that the unit normal to $S_{*}^{ \pm}$coincides with $\pm \boldsymbol{i}_{3}$ while the unit normal to $S_{*}^{\nu}$ takes the form $\boldsymbol{n}=\boldsymbol{\nu}$, where $\boldsymbol{\nu}$ is the unit normal to the plane curve $\mathcal{C}_{*}=\partial \mathcal{M}_{*}, \boldsymbol{\nu} \cdot \boldsymbol{i}_{3}=0$. Considering the identities

$$
\int_{V_{*}}(\ldots) \mathrm{d} V=\int_{-h / 2}^{h / 2} \int_{\mathcal{M}_{*}}(\ldots) \mathrm{d} A \mathrm{~d} z, \quad \int_{S_{*}^{\nu}}(\ldots) \mathrm{d} A=\int_{-h / 2}^{h / 2} \int_{\mathcal{C}_{*}}(\ldots) \mathrm{d} s \mathrm{~d} z
$$

and Eq. (18) $)_{1}$ we derive the relation for the total force vector acting on $V_{*}$

$$
\mathcal{F}^{*}=\int_{\mathcal{M}_{*}}\langle\rho \boldsymbol{f}\rangle \mathrm{d} A+\int_{\mathcal{C}_{*}} \boldsymbol{\nu} \cdot\langle\boldsymbol{\sigma}\rangle \mathrm{d} s+\int_{\mathcal{S}_{*}^{+}} \boldsymbol{t}^{+} \mathrm{d} A+\int_{\mathcal{S}_{*}^{-}} \boldsymbol{t}^{-} \mathrm{d} A, \quad\langle(\ldots)\rangle=\int_{-h / 2}^{h / 2}(\ldots) \mathrm{d} z .
$$

\footnotetext{
${ }^{2}$ http://www.mathematik.tu-darmstadt.de/fbereiche/analysis/pde/staff/neff/patrizio/Home.html
} 
The position vector $\boldsymbol{r}$ of the plate-like body takes the form $\boldsymbol{r}=\boldsymbol{x}+z \boldsymbol{i}_{3}$, where $\boldsymbol{x}$ is the position vector of $\mathcal{M}$. Hence, the total couple is given by

$$
\begin{aligned}
\mathcal{M}^{*}= & \int_{V_{*}} \rho\left[\left(\boldsymbol{x}+z \boldsymbol{i}_{3}\right) \times \boldsymbol{f}+\boldsymbol{\ell}\right] \mathrm{d} V+\int_{S_{*}^{\nu}}\left[\left(\boldsymbol{x}+z \boldsymbol{i}_{3}\right) \times \boldsymbol{t}+\boldsymbol{m}\right] \mathrm{d} A \\
& +\int_{S_{*}^{+}}\left[\left(\boldsymbol{x}+\frac{h}{2} \boldsymbol{i}_{3}\right) \times \boldsymbol{t}^{+}+\boldsymbol{m}^{+}\right] \mathrm{d} A+\int_{S_{*}^{-}}\left[\left(\boldsymbol{x}-\frac{h}{2} \boldsymbol{i}_{3}\right) \times \boldsymbol{t}^{-}+\boldsymbol{m}^{-}\right] \mathrm{d} A \\
= & \int_{\mathcal{M}_{*}}\left[\langle\rho \boldsymbol{\ell}\rangle+\boldsymbol{x} \times\langle\rho \boldsymbol{f}\rangle+\boldsymbol{i}_{3} \times\langle\rho z \boldsymbol{f}\rangle\right] \mathrm{d} A+\int_{S_{*}^{\nu}}\left[\boldsymbol{m}-\boldsymbol{\nu} \cdot \boldsymbol{\sigma} \times \boldsymbol{x}-z \boldsymbol{\nu} \cdot \boldsymbol{\sigma} \times \boldsymbol{i}_{3}\right] \mathrm{d} A \\
& +\int_{S_{*}^{+}}\left(\boldsymbol{x} \times \boldsymbol{t}^{+}+\frac{h}{2} \boldsymbol{i}_{3} \times \boldsymbol{t}^{+}+\boldsymbol{m}^{+}\right) \mathrm{d} A+\int_{S_{*}^{-}}\left(\boldsymbol{x} \times \boldsymbol{t}^{-}-\frac{h}{2} \boldsymbol{i}_{3} \times \boldsymbol{t}^{-}+\boldsymbol{m}^{-}\right) \mathrm{d} A .
\end{aligned}
$$

Taking into account the identity

$$
\int_{\mathcal{S}_{*}^{ \pm}}(\ldots) \mathrm{d} A=\int_{\mathcal{M}_{*}}(\ldots) \mathrm{d} A
$$

we reduce Eq. (27) to the following form

$$
\begin{aligned}
\mathcal{M}^{*}= & \int_{\mathcal{M}_{*}}\left[\langle\rho \boldsymbol{\ell}\rangle+\boldsymbol{x} \times\langle\rho \boldsymbol{f}\rangle+\boldsymbol{i}_{3} \times\langle\rho z \boldsymbol{f}\rangle+\boldsymbol{x} \times\left(\boldsymbol{t}^{+}+\boldsymbol{t}^{-}\right)+\frac{h}{2} \boldsymbol{i}_{3} \times\left(\boldsymbol{t}^{+}-\boldsymbol{t}^{-}\right)+\boldsymbol{m}^{+}+\boldsymbol{m}^{-}\right] \mathrm{d} A \\
& +\int_{\mathcal{C}_{*}^{\nu}}\left[\boldsymbol{\nu} \cdot\langle\boldsymbol{\mu}\rangle-\boldsymbol{\nu} \cdot\langle\boldsymbol{\sigma}\rangle \times \boldsymbol{x}-\boldsymbol{\nu} \cdot\left\langle z \boldsymbol{\sigma} \times \boldsymbol{i}_{3}\right\rangle\right] \mathrm{d} s .
\end{aligned}
$$

Introducing the notations

$$
\boldsymbol{q}=\langle\rho \boldsymbol{f}\rangle+\boldsymbol{t}^{+}+\boldsymbol{t}^{-}, \quad \boldsymbol{c}=\langle\rho \boldsymbol{\ell}\rangle+\boldsymbol{m}^{+}+\boldsymbol{m}^{-}+\boldsymbol{i}_{3} \times\langle\rho z \boldsymbol{f}\rangle+\frac{h}{2} \boldsymbol{i}_{3} \times\left(\boldsymbol{t}^{+}-\boldsymbol{t}^{-}\right)
$$

we transform Eqs. (26) and (28) to the relations

$$
\begin{aligned}
\mathcal{F}^{*} & =\int_{\mathcal{M}_{*}} \boldsymbol{q} \mathrm{d} A+\int_{\mathcal{C}_{*}} \boldsymbol{\nu} \cdot\langle\boldsymbol{\sigma}\rangle \mathrm{d} s \\
\mathcal{M}^{*} & =\int_{\mathcal{M}_{*}}[\boldsymbol{x} \times \boldsymbol{q}+\boldsymbol{c}] \mathrm{d} A+\int_{\mathcal{C}_{*}}\left[\boldsymbol{\nu} \cdot\langle\boldsymbol{\mu}\rangle-\boldsymbol{\nu} \cdot\left\langle z \boldsymbol{\sigma} \times \boldsymbol{i}_{3}\right\rangle-\boldsymbol{\nu} \cdot\langle\boldsymbol{\sigma}\rangle \times \boldsymbol{x}\right] \mathrm{d} A .
\end{aligned}
$$

The comparison of Eqs. (30) and (1) leads to the determination of the surface stress tensor and the surface couple stress tensors by the following relations

$$
\mathbf{T}=\langle\mathbf{A} \cdot \boldsymbol{\sigma}\rangle, \quad \mathbf{M}=\langle\mathbf{A} \cdot \boldsymbol{\mu}\rangle-\left\langle\mathbf{A} \cdot z \boldsymbol{\sigma} \times \boldsymbol{i}_{3}\right\rangle .
$$

If $\boldsymbol{\mu}=\mathbf{0}$ then Eqs. (31) reduce to the definition of the stress resultant tensor and couple resultant stress tensors given, for example in $[8,42,73]$. Let us note that from Eq. $(31)_{2}$ it follows that the transverse shear moments $M_{\alpha 3}$ depend only upon the couple stress tensor $\boldsymbol{\mu}$. Indeed, $\mathbf{M} \cdot \boldsymbol{i}_{3}=\left\langle\mathbf{A} \cdot \boldsymbol{\mu} \cdot \boldsymbol{i}_{3}\right\rangle$.

Thus, if one assumes the definitions (29) and (31) for the surface loads and the surface stress measures then the twodimensional balance equations (1) are the exact consequence of the three-dimensional equilibrium conditions (17). On the other hand, Eqs. (1) and (2) are the exact two-dimensional equilibrium conditions expressed in the terms of the surface stresses and couples derived using the direct approach. This means that the presented in Sect. 2 theory of micropolar plates is the general linear theory of plates based on the concept of forces and couples acting on a deformable plane surface embedded in three-dimensional space. 


\section{Micropolar plate's constitutive equations derived by the through-the-thickness integration procedure}

In this section we calculate the tensors $\mathbf{T}$ and $\mathbf{M}$ using Eqs. (31). Let us assume that the plate-like body is homogeneous in $z$-direction, i.e. that the micropolar moduli $\lambda, \mu, \kappa, \alpha, \beta, \gamma$ do not depend on $z$.

In the "standard" theory of plates the assumption $\sigma_{33}=0$ is applied, see [70]. Using this assumption from Eq. (23) $)_{1}$ we obtain that

$$
\varepsilon_{33}=-\frac{\lambda}{\lambda+2 \mu+\kappa}\left(\varepsilon_{11}+\varepsilon_{22}\right) .
$$

Hence, Eq. (23) $)_{1}$ transforms to

$$
\boldsymbol{\sigma}=\tilde{\lambda} \mathbf{I}\left(\varepsilon_{11}+\varepsilon_{22}\right)+(\mu+\kappa) \boldsymbol{\varepsilon}+\mu \boldsymbol{\varepsilon}^{T}=\tilde{\lambda} \mathbf{I}\left(\varepsilon_{11}+\varepsilon_{22}\right)+(\mu+\kappa) \nabla \boldsymbol{u}+\mu(\nabla \boldsymbol{u})^{T}+\kappa \mathbf{I} \times \boldsymbol{\vartheta},
$$

where $\tilde{\lambda}=\frac{\lambda(2 \mu+\kappa)}{\lambda+2 \mu+\kappa}$. The following relations hold true

$$
\nabla_{s}=\mathbf{A} \cdot \nabla, \quad \mathbf{A} \times \boldsymbol{\vartheta}=\boldsymbol{\vartheta} \times \boldsymbol{i}_{3} \otimes \boldsymbol{i}_{3}+\vartheta_{3} \mathbf{A} \times \boldsymbol{i}_{3}=\boldsymbol{\vartheta}_{s} \times \boldsymbol{i}_{3} \otimes \boldsymbol{i}_{3}+\vartheta_{3} \mathbf{A} \times \boldsymbol{i}_{3},
$$

$$
\mathbf{A} \cdot \nabla \boldsymbol{u}=\nabla_{s} \boldsymbol{u}_{s}+\left(\nabla_{s} u_{3}\right) \otimes \boldsymbol{i}_{3}, \quad \mathbf{A} \cdot(\nabla \boldsymbol{u})^{T}=\left(\nabla_{s} \boldsymbol{u}_{s}\right)^{T}+\boldsymbol{u}_{s, 3} \otimes \boldsymbol{i}_{3},
$$

where $\boldsymbol{u}_{s} \equiv \mathbf{A} \cdot \boldsymbol{u}, \boldsymbol{\vartheta}_{s} \equiv \mathbf{A} \cdot \vartheta$. Thus, we obtain

$$
\begin{aligned}
\mathbf{A} \cdot \boldsymbol{\sigma}= & \tilde{\lambda} \mathbf{A} \nabla_{s} \cdot \boldsymbol{u}_{s}+(\mu+\kappa)\left(\nabla_{s} \boldsymbol{u}_{s}+\vartheta_{3} \mathbf{A} \times \boldsymbol{i}_{3}\right)+(\mu+\kappa)\left(\nabla_{s} u_{3}+\boldsymbol{\vartheta} \times \boldsymbol{i}_{3}\right) \otimes \boldsymbol{i}_{3} \\
& +\mu\left(\nabla_{s} \boldsymbol{u}_{s}+\vartheta_{3} \mathbf{A} \times \boldsymbol{i}_{3}\right)^{T}+\mu \boldsymbol{u}_{s, 3} \otimes \boldsymbol{i}_{3}-\mu \boldsymbol{\vartheta} \times \boldsymbol{i}_{3} \otimes \boldsymbol{i}_{3} .
\end{aligned}
$$

Introducing the notations

$$
h \tilde{\boldsymbol{\epsilon}}=\nabla_{s}\langle\boldsymbol{u}\rangle+\mathbf{A} \times\langle\boldsymbol{\vartheta}\rangle, \quad \tilde{\boldsymbol{\epsilon}}_{\|}=\tilde{\boldsymbol{\epsilon}} \cdot \mathbf{A},
$$

one gets the relation

$$
\mathbf{T}=\tilde{\lambda} h \mathbf{A} \operatorname{tr} \tilde{\boldsymbol{\epsilon}}+(\mu+\kappa) h \tilde{\boldsymbol{\epsilon}}_{\|}+\mu h \tilde{\boldsymbol{\epsilon}}_{\|}^{T}+(\mu+\kappa) h(\tilde{\boldsymbol{\epsilon}} \cdot \boldsymbol{n}) \otimes \underline{\boldsymbol{n}-\mu h\left[\langle\boldsymbol{\vartheta}\rangle \times \boldsymbol{i}_{3}-\left\langle\boldsymbol{u}_{s, 3}\right\rangle\right] \otimes \boldsymbol{i}_{3} .}
$$

The comparison of Eqs. (34) and Eq. (12) shows, that they have the same structure and that there is only one difference the underlined terms in Eq. (34). If we assume that the following relation holds true

$$
\langle\boldsymbol{\vartheta}\rangle \times \boldsymbol{i}_{3}=\left\langle\boldsymbol{u}_{s, 3}\right\rangle,
$$

Eqs. (34) and (12) coincide up to notations.

Let us consider Eq. (31) 2 . M consists of two terms. The first one is given by the relation

$$
\langle\mathbf{A} \cdot \boldsymbol{\mu}\rangle=\alpha \mathbf{A}\left(\nabla_{s} \cdot\left\langle\boldsymbol{\vartheta}_{s}\right\rangle+\left\langle\vartheta_{3,3}\right\rangle\right)+\beta\left[\left(\nabla_{s}\left\langle\boldsymbol{\vartheta}_{s}\right\rangle\right)^{T}+\left\langle\boldsymbol{\vartheta}_{s, 3}\right\rangle \otimes \boldsymbol{i}_{3}\right]+\gamma\left(\nabla_{s}\left\langle\boldsymbol{\vartheta}_{s}\right\rangle+\nabla_{s}\left\langle\vartheta_{3}\right\rangle \otimes \boldsymbol{i}_{3}\right) .
$$

Using Eq. (33) we obtain the relation

$$
\mathbf{A} \cdot \boldsymbol{\sigma} \times \boldsymbol{i}_{3}=\tilde{\lambda} \mathbf{A} \times \boldsymbol{i}_{3} \nabla_{s} \cdot \boldsymbol{u}_{s}+(\mu+\kappa)\left(\nabla_{s} \boldsymbol{u}_{s}\right) \times \boldsymbol{i}_{3}+\mu\left(\nabla_{s} \boldsymbol{u}_{s}\right)^{T} \times \boldsymbol{i}_{3}-\kappa \vartheta_{3} \mathbf{A},
$$

and then the second term in Eq. $(31)_{2}$ takes the form

$$
\left\langle\mathbf{A} \cdot z \boldsymbol{\sigma} \times \boldsymbol{i}_{3}\right\rangle=\tilde{\lambda} \mathbf{A} \times \boldsymbol{i}_{3} \nabla_{s} \cdot\left\langle z \boldsymbol{u}_{s}\right\rangle+(\mu+\kappa) \nabla_{s}\left\langle z \boldsymbol{u}_{s}\right\rangle \times i_{3}+\mu\left(\nabla_{s}\left\langle z \boldsymbol{u}_{s}\right\rangle\right)^{T} \times \boldsymbol{i}_{3}-\kappa\left\langle z \vartheta_{3}\right\rangle \mathbf{A} .
$$

Introducing the vector $\boldsymbol{\psi}=-\left\langle z \boldsymbol{u}_{s}\right\rangle \times \boldsymbol{i}_{3}$, Eq. (37) may be transformed to the following form

$$
\left\langle\mathbf{A} \cdot z \boldsymbol{\sigma} \times \boldsymbol{i}_{3}\right\rangle=-\mu \mathbf{A} \nabla_{s} \cdot \boldsymbol{\psi}-(\mu+\kappa-\tilde{\lambda}) \nabla_{s} \boldsymbol{\psi}-(\tilde{\lambda}-\mu)\left(\nabla_{s} \boldsymbol{\psi}\right)^{T}-\kappa\left\langle z \vartheta_{3}\right\rangle \mathbf{A} .
$$

Hence, using Eqs. (36) and (38) we obtain the expression for $\mathbf{M}$

$$
\mathbf{M}=\alpha \mathbf{A}\left(\nabla_{s} \cdot\left\langle\boldsymbol{\vartheta}_{s}\right\rangle+\left\langle\vartheta_{3,3}\right\rangle\right)+\beta\left[\left(\nabla_{s}\left\langle\boldsymbol{\vartheta}_{s}\right\rangle\right)^{T}+\left\langle\boldsymbol{\vartheta}_{s, 3}\right\rangle \otimes \boldsymbol{i}_{3}\right]+\gamma\left(\nabla_{s}\left\langle\boldsymbol{\vartheta}_{s}\right\rangle+\nabla_{s}\left\langle\vartheta_{3}\right\rangle \otimes \boldsymbol{i}_{3}\right)
$$




$$
\begin{aligned}
& +\mu \mathbf{A} \nabla_{s} \cdot \boldsymbol{\psi}+(\mu+\kappa-\tilde{\lambda}) \nabla_{s} \boldsymbol{\psi}+(\tilde{\lambda}-\mu)\left(\nabla_{s} \boldsymbol{\psi}\right)^{T}+\kappa\left\langle z \vartheta_{3}\right\rangle \mathbf{A} \\
= & \mathbf{A} \nabla_{s} \cdot\left(\alpha\left\langle\boldsymbol{\vartheta}_{s}\right\rangle+\mu \boldsymbol{\psi}\right)+\left[\nabla_{s}\left(\beta\left\langle\boldsymbol{\vartheta}_{s}\right\rangle+(\tilde{\lambda}-\mu) \boldsymbol{\psi}\right)\right]^{T} \\
& +\nabla_{s}\left(\gamma\left\langle\boldsymbol{\vartheta}_{s}\right\rangle+(\mu+\kappa-\tilde{\lambda}) \boldsymbol{\psi}\right)+\gamma \nabla_{s}\left\langle\vartheta_{3}\right\rangle \otimes \boldsymbol{i}_{3} \\
& +\underline{\mathbf{A}\left(\alpha\left\langle\vartheta_{3,3}\right\rangle+\kappa\left\langle z \vartheta_{3}\right\rangle\right)+\beta\left\langle\boldsymbol{\vartheta}_{s, 3}\right\rangle \otimes \boldsymbol{i}_{3}} .
\end{aligned}
$$

One can see that Eq. (39) is inconsistent with Eq. (13), in general. The structure of $\mathbf{M}$ in Eq. (39) differs from Eq. (13) by the underlined terms. For consistency we assume that the following relations hold true

$$
\left\langle\vartheta_{3,3}\right\rangle=0, \quad\left\langle\boldsymbol{\vartheta}_{s, 3}\right\rangle=\mathbf{0}, \quad\left\langle z \vartheta_{3}\right\rangle=0 .
$$

For example, Eqs. (40) are satisfied if $\vartheta$ is a even function of $z$.

To introduce the effective wryness tensor $\tilde{\kappa}$ let us assume that

$$
\left\langle\boldsymbol{\vartheta}_{s}\right\rangle=-\frac{1}{l^{2}}\left\langle z \boldsymbol{u}_{s}\right\rangle \times \boldsymbol{i}_{3},
$$

where $l$ is a parameter which has the dimension of length. Then $\mathbf{M}$ takes the form

$$
\mathbf{M}=\left(\alpha / l^{2}+\mu\right) \mathbf{A} \nabla_{s} \cdot \boldsymbol{\psi}+\left(\beta / l^{2}+\tilde{\lambda}-\mu\right)\left(\nabla_{s} \boldsymbol{\psi}\right)^{T}+\left(\gamma / l^{2}+\mu+\kappa-\tilde{\lambda}\right) \nabla_{s} \boldsymbol{\psi}+\gamma h \nabla_{s} \tilde{\theta}_{3} \otimes \boldsymbol{i}_{3}
$$

where $h \tilde{\theta}_{3}=\left\langle\vartheta_{3}\right\rangle$.

Note that we assumed the relations (35) and (41) which may not be satisfied simultaneously, in general. The assumptions (35), (40), and (41) are satisfied identically if one applies the following approximation of $\boldsymbol{u}$ and $\vartheta$ with $l^{2}=h^{2} / 12$

$$
\boldsymbol{u}(x, y, z)=\boldsymbol{v}(x, y)-z \phi(x, y), \quad \boldsymbol{\vartheta}=\boldsymbol{\phi}(x, y) \times \boldsymbol{i}_{3}+\vartheta_{3}(x, y) \boldsymbol{i}_{3}, \quad \boldsymbol{\phi} \cdot \boldsymbol{i}_{3}=0 .
$$

This means that the couple stress tensor $\boldsymbol{\mu}$ does not depend on $z$, while the stress tensor $\boldsymbol{\sigma}$ depends on $z$ linearly as in $[24,25]$. But the approximation (43) is more restrictive than used by Eringen [24,25]. The analogous to (43) approximation was assumed in [29] where the theory of the linear three-dimensional Cosserat pseudo-continuum was used [44,52]. Relations (43) do not coincide with the constraint of the Cosserat pseudo-continuum because here $\boldsymbol{\vartheta} \neq \frac{1}{2} \nabla \times \boldsymbol{u}$. Equations (43) state that only in-plane components of the microrotation vector coincide with in-plane components of the macrorotation vector $\frac{1}{2} \nabla \times \boldsymbol{u}$.

Assuming Eqs. (43), the tensors $\mathbf{T}$ and $\mathbf{M}$ take the form

$$
\begin{aligned}
\mathbf{T}= & \tilde{\lambda} h \mathbf{A} \nabla_{s} \cdot \boldsymbol{v}+(\mu+\kappa) h \nabla_{s} \boldsymbol{v}_{s}+\mu h\left(\nabla_{s} \boldsymbol{v}_{s}\right)^{T}+\kappa h \vartheta_{3} \mathbf{A} \times i_{3}+(\mu+\kappa) h\left[\nabla_{s}\left(\boldsymbol{v} \cdot \boldsymbol{i}_{3}\right)-\boldsymbol{\phi}\right] \otimes \boldsymbol{i}_{3} \\
= & \tilde{\lambda} h \mathbf{A} \operatorname{tr} \tilde{\boldsymbol{\epsilon}}+(\mu+\kappa) h \tilde{\boldsymbol{\epsilon}}_{\|}+\mu h \tilde{\boldsymbol{\epsilon}}_{\|}^{T}+(\mu+\kappa) h(\tilde{\boldsymbol{\epsilon}} \cdot \boldsymbol{n}) \otimes \boldsymbol{n}, \\
\mathbf{M}= & \left\{\left(\tilde{\lambda} \frac{h^{3}}{12}-\beta h\right) \mathbf{A} \nabla_{s} \cdot \boldsymbol{\phi}+\left(\mu \frac{h^{3}}{12}-\alpha h\right)\left(\nabla_{s} \boldsymbol{\phi}\right)^{T}+\left[(\mu+\kappa) \frac{h^{3}}{12}+(\alpha+\beta+\gamma) h\right] \nabla_{s} \boldsymbol{\phi}\right\} \\
& +\gamma h \nabla_{s} \vartheta_{3} \otimes \boldsymbol{i}_{3} \\
= & \left(\alpha h-\mu \frac{h^{3}}{12}\right) \mathbf{A t r} \tilde{\boldsymbol{\kappa}}+\left[\gamma h+(2 \mu+\kappa+\tilde{\lambda}) \frac{h^{3}}{12}\right] \tilde{\boldsymbol{\kappa}}_{\|}+\left[\beta-\tilde{\lambda} \frac{h^{3}}{12}\right] \tilde{\boldsymbol{\kappa}}_{\|}^{T} \\
& +\gamma h \tilde{\boldsymbol{\kappa}} \cdot \boldsymbol{n} \otimes \boldsymbol{n}, \\
\tilde{\boldsymbol{\epsilon}}= & \nabla_{s} \boldsymbol{v}+\mathbf{A} \times \boldsymbol{\theta}=\nabla_{s} \boldsymbol{v}+\vartheta_{3} \mathbf{A} \times \boldsymbol{i}_{3}-\boldsymbol{\phi} \otimes \boldsymbol{i}_{3}, \quad \boldsymbol{\theta}=\boldsymbol{\vartheta}, \quad \tilde{\boldsymbol{\kappa}}=\nabla_{s} \boldsymbol{\theta} .
\end{aligned}
$$

As a result the effective stiffness parameters are given by the relations

$$
\begin{aligned}
& \alpha_{1}=\tilde{\lambda} h \equiv \frac{\lambda(2 \mu+\kappa)}{\lambda+2 \mu+\kappa} h, \quad \alpha_{2}=\mu h, \quad \alpha_{3}=(\mu+\kappa) h, \quad \alpha_{4}=(\mu+\kappa) h, \\
& \beta_{1}=\alpha h-\mu \frac{h^{3}}{12}, \quad \beta_{2}=\beta h-\tilde{\lambda} \frac{h^{3}}{12}, \quad \beta_{3}=\gamma h+(2 \mu+\kappa+\tilde{\lambda}) \frac{h^{3}}{12}, \quad \beta_{4}=\gamma h .
\end{aligned}
$$

The in-plane stiffness parameters $\alpha_{1}, \alpha_{3}, \alpha_{3}$, and the transverse shear stiffness $\alpha_{4}$ depend linearly on $h$. The dependence of $\beta_{i}, i=1, \ldots 4$ on $h$ is more complicated. From Eqs. (45) it is evident that $\beta_{i}$ have the linear asymptotes when $h$ tends to zero: $\beta_{i} \sim h$. The considered case differs from the case of Kirchhoff's plate or Reissner's plate when $\beta_{i} \sim h^{3}$. 
To illustrate the dependence of the elastic stiffness on the thickness let us consider the bending stiffness of a micropolar plate which is given by

$$
D=(2 \mu+\kappa+\tilde{\lambda}) \frac{h^{3}}{12}+\gamma h .
$$

Introducing the technical constants

$$
G=\frac{2 \mu+\kappa}{2}, \quad \nu=\frac{\lambda}{2 \lambda+2 \mu+\kappa}, \quad l_{b}^{2}=\frac{\gamma}{2(2 \mu+\kappa)},
$$

where $G$ is the shear modulus, $\nu$ is the Poisson ratio, $l_{b}$ is the characteristic length under bending, see [25], we obtain the expression

$$
D=\frac{G h^{3}}{12(1-\nu)}\left[1+2 \frac{l_{b}^{2}}{h^{2}}\right]
$$

The dependence $D / \widetilde{D}$ on $h$ is given in Fig. 3, where $\widetilde{D}=\frac{G h^{3}}{12(1-\nu)}$ is the value of the bending stiffness used by Eringen [24,25]. From Fig. 3 it is seen that the micropolar properties are inessential if $h>2 l_{b}$.

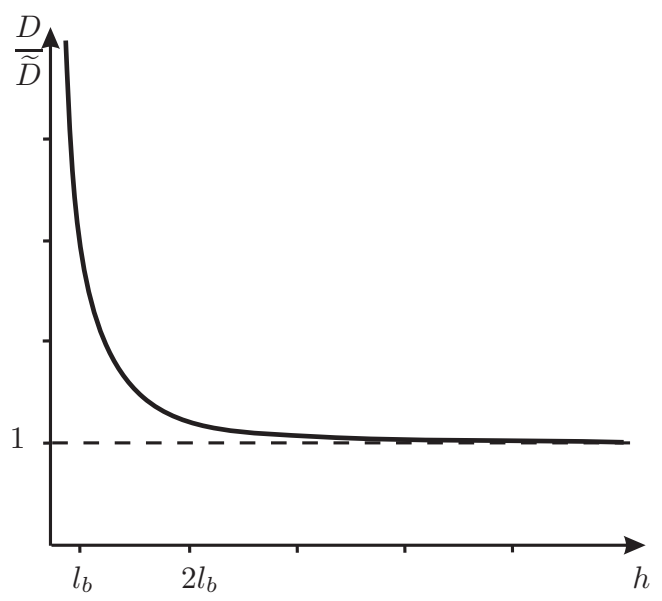

Fig. 3 Dimensionless bending stiffness $D / \widetilde{D}$ vs. the dimensionless thickness $h / l_{b}$.

It should be noted that the formulation procedure of the two-dimensional constitutive equations applied in this section is the same like in the case of the Kirchhoff theory. It is well-known that the Kirchhoff theory cannot predict the transverse shear stiffness in a right manner. In the Kirchhoff theory the stiffness parameters are undetermined. An analogous situation we have here if we try to determine the stiffness related to the drilling moment. The value $\beta_{4}$ can be obtained only with the help of the values of the micropolar parameters. In addition, the value of the shear stiffness $\alpha_{4}=(\mu+\kappa) h$ predicted here in the classical case is equal to the shear correction factor 1 , and one gets an overestimation in comparison with Reissner's $5 / 6$ or Mindlin's $\pi^{2} / 12$. Such over- or underestimation of the stiffness corresponding to the drilling moment is also possible with the used approach. But by this value one can see the influence of the micropolar properties on the stiffness parameters. In particular, Eqs. (45) and (46) demonstrate the size-effect, which is present in the micropolar elasticity, see [25, 38-40]. The analysis of the formulas (45) and (46) show that the influence of the micropolar properties is essential if the thickness of a plate $h$ has the same value as the characteristic length of the microstructure of a material. This means that the influence of the micropolar properties may be significant for very thin plates.

The equilibrium equations for the forces in the present theory $(2)_{1}$ are the same like in Eringen's theory if the equilibrium is assumed, see Eqs. (5.27.5) and (5.27.6) in [25]. The equilibrium equations for the moments $(2)_{2}$ are different from (5.27.7)-(5.27.9) in [25]. The differences can be obtained comparing the structure, in addition the number of unknowns is different. Eringen used the linear approximation

$$
\boldsymbol{u}(x, y, z)=\boldsymbol{v}(x, y)-z \phi(x, y), \quad \boldsymbol{\vartheta}(x, y, z)=\boldsymbol{\xi}(x, y), \quad \boldsymbol{\phi} \cdot \boldsymbol{i}_{3}=0,
$$

with two vector fields $\phi(x, y), \boldsymbol{\xi}(x, y)$. Eringen's plate equations and the equations of the present theory are the same in the case if one can assume the in-plane behavior only: $\boldsymbol{v}=v_{1}(x, y) \boldsymbol{i}_{1}+v_{2}(x, y) \boldsymbol{i}_{2}, \boldsymbol{\theta}=\theta_{3}(x, y) \boldsymbol{i}_{3}$. The constitutive equations in [25] are written in terms of the following stress and couple stress resultants

$$
\mathbf{T}=\frac{1}{h}\langle\boldsymbol{\sigma}\rangle, \quad \overline{\mathbf{M}}=\frac{1}{h}\langle\boldsymbol{\mu}\rangle, \quad \mathbf{M}=\frac{1}{h}\langle z \boldsymbol{\sigma}\rangle,
$$


which are expressed in terms of $\mathbf{v}, \phi$, and $\boldsymbol{\xi}$.

Gevorkyan [29] obtained the constitutive equations for the $\mathbf{T}$ and $\mathbf{M}$ in the form (12), (13) with constants $\alpha_{1}, \alpha_{2}, \alpha_{3}$ coinciding with (16) but with $\alpha_{4}=0$. The drilling moments $M_{\alpha 3}$ are generated by the couple stress tensor $\boldsymbol{\mu}$ only. The constants $\beta_{i}$ have different values then (16) or (45).

Reissner [63] introduced the stress resultants $\mathbf{T}$, the force-stress couple $\mathbf{M}^{\sigma}$, and the moment-stress resultant $\mathbf{M}^{\mu}$

$$
\mathbf{T}=\langle\boldsymbol{\sigma}\rangle, \quad \mathbf{M}^{\sigma}=\langle\boldsymbol{\mu}\rangle, \quad \mathbf{M}^{\mu}=\langle z \boldsymbol{\sigma}\rangle
$$

taking into account the transverse shear forces and the drilling moment. He used the linear approximation in $z$ coordinate for the stresses acting in the three-dimensional plate-like body. The constitutive equations in the Reissner theory are expressed in the distinct rotational strain measures corresponding to $\mathbf{M}^{\sigma}$ and $\mathbf{M}^{\mu}$.

Comparing the present theory with Reissner theory $[59,60]$, our system of equations contains an additional parameter the component of the rotation vector $\theta_{3}$. In Reissner's theory it is assumed that $\theta_{3}=0$. Here the sixth equilibrium equation (5) $)_{6}$ is nontrivial since in the six-parameter theory the components of the moment tensor $M_{13}$ and $M_{23}$ are included. In Reissner's theory $M_{13}=M_{23}=0$. From this point of view the transition from the present theory to Reissner's theory can be realized assuming that the components of the moment tensor $M_{13}$ and $M_{23}$ and the drilling moment $c_{3}$ are equal zero. In addition, the shear correction factor should be taken into account for the transverse shear stiffness $\alpha_{4}$.

It should be noted that the constitutive equations presented here and the effective stiffness parameters are based on various assumptions on the inner plate structure and the deformations:

- The plate is homogeneous.

- The normal stress $\sigma_{33}=0$. This means that like in the Kirchhoff theory the normal stresses $\sigma_{33}$ can be neglected in comparison with the other stress tensor components.

- $\langle\boldsymbol{\vartheta}\rangle \times \boldsymbol{i}_{3}=\left\langle\boldsymbol{u}_{s, 3}\right\rangle,\langle\boldsymbol{\vartheta}\rangle \times \boldsymbol{i}_{3}=\frac{1}{l^{2}}\left\langle z \boldsymbol{u}_{s}\right\rangle$.

- $\left\langle\boldsymbol{\vartheta}_{, 3}\right\rangle=0,\left\langle z \vartheta_{3}\right\rangle=0$.

If we neglect all hypotheses or only a part of them one gets another effective stiffness parameters for the micropolar plate. This conclusion is especially related to the drilling stiffness. An alternative approaches one can apply:

- The comparison of some boundary-value problems of the three-dimensional micropolar theory of elasticity (for example, tension and bending problem for a parallelepiped, shear and torsion of a prism) with the conjugated twodimensional problems. In the case of a nonpolar continuum this was demonstrated in $[3,4,6,73]$. In these papers, for example, the nonclassical values for the transverse shear coefficient were obtained.

- The consistent approximation of the energy of the micropolar continuum. For the case of a nonpolar continuum this was realized in $[56,57]$.

\section{Example of the elastic stiffness parameters for the micropolar plates}

As an example let us consider the elastic stiffness parameters using the experimental data presented by Lakes [38, 40], see Table 1. Using the size-effect, Lakes established that some foams demonstrate the micropolar elastic behavior. In Table 1 PS is a low-density polystyrene closed-cell foam, while PU is a high-density rigid polyurethane closed-cell foam.

The corresponding elastic stiffness parameters are presented in Table 2. Let us note that the moment stiffness $\beta_{4}$ is less than other bending stiffness parameters. Here we also present the elastic stiffness parameters if one neglects the micropolar properties, i.e. if $\kappa=\alpha=\beta=\gamma=0$. The the corresponding columns in Table 2 are marked by the index *. It is seen that the micropolar constants play a role if the thickness of the plate is of the order of $\sim 10^{-2} \mathrm{~m}$ for the first foam and $\sim 10^{-3} \mathrm{~m}$ for the second one. On the other hand it means that the plate thickness is approximately equal to the size of the foam cell. The application of the plate theory to such structures is under consideration.

\section{Conclusions}

We discuss here the general six-parametric or micropolar linear plate theory with two vector fields of the translations and of the rotations as the independent kinematic variables. Within the proposed theory one may take into account an external surface drilling moment. Within the direct approach the equilibrium conditions and the constitutive equations are formulated as for the two-dimensional Cosserat continuum. This means that we consider a plate as a deformable surface with three orthonormal directors attached at any point of the surface. We are restricted by the case of infinitesimal translations and rotations. The equilibrium equations are deduced from the two-dimensional integral equilibrium conditions which state that 
Table 1 Micropolar constants for two materials [38, 40].

\begin{tabular}{llll}
\hline & & Foam, PU & Foam, PS \\
\hline Shear modulus, MPa & $G=\frac{2 \mu+\kappa}{2}$ & 1.1 & 104 \\
Poisson's ratio & $\nu=\frac{\lambda}{2 \lambda+2 \mu+\kappa}$ & 0.07 & 0.4 \\
Characteristic length (torsion), $\mathrm{mm}$ & $l_{t}=\sqrt{\frac{\beta+\gamma}{2 \mu+\kappa}}$ & 3.8 & 0.62 \\
Characteristic length (bending), $\mathrm{mm}$ & $l_{b}=\sqrt{\frac{\gamma}{2(2 \mu+\kappa)}}$ & 5.0 & 0.33 \\
Coupling number & $N^{2}=\frac{\kappa}{2 \mu+\kappa}$ & 0.09 & 0.04 \\
Polar ratio & $\Psi=\frac{\beta+\gamma}{\alpha+\beta+\gamma}$ & 1.5 & 1.5 \\
\hline
\end{tabular}

Table 2 Effective stiffness of a plate made of different porous materials ( $h$ has the dimension $\mathrm{m}$ ).

\begin{tabular}{l|l|llll}
\hline $\begin{array}{l}\text { Elastic } \\
\text { constants }\end{array}$ & & Foam, PU & Foam, PU * & Foam, PS & Foam, PS * \\
\hline$\alpha_{1}, \mathrm{~N} / \mathrm{m} 10^{6}$ & $\tilde{\lambda} h$ & $0.165 h$ & $0.165 h$ & $138.67 h$ & $138.67 h$ \\
$\alpha_{2}, \mathrm{~N} / \mathrm{m} 10^{6}$ & $\mu h$ & $1.001 h$ & $1.1 h$ & $99.84 h$ & $104 h$ \\
$\alpha_{3}, \mathrm{~N} / \mathrm{m} 10^{6}$ & $(\mu+\kappa) h$ & $1.199 h$ & $1.1 h$ & $108.16 h$ & $104 h$ \\
$\alpha_{4}, \mathrm{~N} / \mathrm{m} 10^{6}$ & $(\mu+\kappa) h$ & $1.199 h$ & $1.1 h$ & $108.16 h$ & $104 h$ \\
$\beta_{1}, \mathrm{~N} \cdot \mathrm{m} 10^{6}$ & $\alpha h-\mu \frac{h^{3}}{12}$ & $-2.6 \cdot 10^{-6} h-0.083 h^{3}$ & $-0.092 h^{3}$ & $-6.7 \cdot 10^{-6} h-8.3 h^{3}$ & $-8.67 h^{3}$ \\
$\beta_{2}, \mathrm{~N} \cdot \mathrm{m} 10^{6}$ & $\beta h-\tilde{\lambda} \frac{h^{3}}{12}$ & $-10^{-4} h-0.014 h^{3}$ & $-0.014 h^{3}$ & $-2.5 \cdot 10^{-5} h+11.6 h^{3}$ & $-11.5 h^{3}$ \\
$\beta_{3}, \mathrm{~N} \cdot \mathrm{m} 10^{6}$ & $\gamma h+(2 \mu+\kappa+\tilde{\lambda}) \frac{h^{3}}{12}$ & $1.1 \cdot 10^{-4} h+0.197 h^{3}$ & $0.197 h^{3}$ & $4.5 \cdot 10^{-5} h+28.9 h^{3}$ & $28.8 h^{3}$ \\
$\beta_{4}, \mathrm{~N} \cdot \mathrm{m} 10^{6}$ & $\gamma h$ & $1.1 \cdot 10^{-4} h$ & 0 & $4.5 \cdot 10^{-5} h$ & 0 \\
\hline
\end{tabular}

the total force and the total moment vectors acting on any part of the surface are zero. The linear strain measures $\epsilon$ and $\kappa$ are introduced as work-conjugate to the surface stress tensor $\mathbf{T}$ and the surface couple tensor $\mathbf{M}$, respectively. For the linear isotropic elastic behavior the two-dimensional constitutive equations are presented. These equations are the linear tensor-valued functions of $\epsilon$ and $\kappa$ and contain eight elastic constants, in general. Then, starting from the tree-dimensional linear micropolar elasticity we show that the two-dimensional equilibrium conditions are the exact consequence of the three-dimensional integral balances of momentum and moment of momentum if the introduced surface stress and couple stress tensors are the stress resultant and the moment stress resultant tensors, respectively.

For the homogeneous material and using some special approximations of the displacements and the microrotations of a micropolar plate-like body, we calculated the stress resultant and the moment stress resultant tensors as functions of the averaged strain measures. Hence, on the base of the three-dimensional constitutive equations of a micropolar isotropic material we found the effective stiffness parameters of the plate. The non-homogeneous material properties and determination of the elastic stiffness properties of a micropolar plates are discussed. 
Acknowledgements The authors thank Professor Pal'mov for the fruitful discussions on the problem of the effective stiffness tensors in the micropolar theory of plates during the APM 2008 conference, St. Petersburg, Repino, July, 2008. In addition, the first author was supervised by Prof. Pal'mov during his PhD studies and later. During this period many scientific, personal, and other items have been discussed in a qute nice and friendly atmosphere.

The authors also thank Professor Wojciech Pietraszkiewicz and Dr. habil. Patrizio Neff for the discussions of the results presented in this paper.

The second author was supported by the DAAD program "Forschungsaufenthalte für Hochschullehrer und Wissenschaftler" in 2008.

\section{References}

[1] I. Aganović, J. Tambača, and Z. Tutek, Derivation and justification of the models of rods and plates from linearized threedimensional micropolar elasticity, J. Elast. 84, 131-152 (2006).

[2] H. Altenbach, Eine direkt formulierte lineare Theorie für viskoelastische Platten und Schalen, Ing.-Arch. 58, 215-228 (1988).

[3] H. Altenbach, An alternative determination of transverse shear stiffnesses for sandwich and laminated plates, Int. J. Solids Struct. 37(25), 3503-3520 (2000).

[4] H. Altenbach, On the determination of transverse shear stiffnesses of orthotropic plates, ZAMP 51, 629-649 (2000).

[5] H. Altenbach and V. A. Eremeyev, Analysis of the viscoelastic behavior of plates made of functionally graded materials, ZAMM 88(5), 332-341 (2008).

[6] H. Altenbach and V. A. Eremeyev, Direct approach based analysis of plates composed of functionally graded materials, Arch. Appl. Mech. 78(10), 775-794 (2008).

[7] H. Altenbach and V. A. Eremeyev, On the time-dependent behavior of FGM plates, Key Eng. Mater. 399, 63-70 (2009).

[8] H. Altenbach and P. A. Zhilin, A general theory of elastic simple shells (in Russ.), Uspekhi Mekhaniki 11(4), 107-148 (1988).

[9] H. Altenbach and P. A. Zhilin, The Theory of Simple Elastic Shells, in: Critical Review of the Theories of Plates and Shells, edited by R. Kienzler, H. Altenbach, and I. Ott, Lect. Notes Appl. Comp. Mech. Vol. 16 (Springer, Berlin, 2004), pp. 1-12.

[10] S. A. Ambartsumian, The theory of transverse bending of plates with asymmetric elasticity, Mech. Compos. Mater. 32(1), 30-38 (1996).

[11] S. S. Antman, Nonlinear Problems of Elasticity, 2nd edition (Springer Science Media, New York, 2005).

[12] J. Chróścielewski, J. Makowski, and W. Pietraszkiewicz, Statyka i dynamika powłok wielopłatowych. Nieliniowa teoria i metoda elementów skończonych (Wydawnictwo IPPT PAN, Warszawa, 2004).

[13] E. Cosserat and F. Cosserat, Théorie des corps déformables (Herman et Fils, Paris, 1909).

[14] H. A. Erbay, An asymptotic theory of thin micropolar plates, Int. J. Eng. Sci. 38(13), 1497-1516 (2000).

[15] V. A. Eremeyev, Nonlinear Micropolar Shells: Theory and Applications, in: Shell Structures: Theory and Applications, edited by W. Pietraszkiewicz and C. Szymczak (Taylor \& Francis, London, 2005), pp. 11-18.

[16] V. A. Eremeyev and W. Pietraszkiewicz, The non-linear theory of elastic shells with phase transitions, J. Elast. 74(1), 67-86 (2004).

[17] V. A. Eremeyev and W. Pietraszkiewicz, Local symmetry group in the general theory of elastic shells, J. Elast. 85(2), 125-152 (2006).

[18] V. A. Eremeyev and L. M. Zubov, On constitutive inequalities in nonlinear theory of elastic shells, ZAMM 87(2), 94-101 (2007).

[19] V. A. Eremeyev and L. M. Zubov, Mechanics of Elastic Shells (in Russ.) (Nauka, Moscow, 2008).

[20] J. L. Ericksen, Wave propagation in thin elastic shells, J. Elast. 43(3), 167-178 (1971).

[21] J. L. Ericksen, The simplest problems for elastic Cosserat surfaces, J. Elast. 2(2), 101-107 (1972).

[22] J. L. Ericksen, Plane infinitesimal waves in homogeneous elastic plates, J. Elast. 3(3), 161-167 (1973).

[23] J. L. Ericksen and C. Truesdell, Exact tbeory of stress and strain in rods and shells, Arch. Rat. Mech. Anal. 1(1), 295-323 (1958).

[24] A. C. Eringen, Theory of micropolar plates, ZAMP 18(1), 12-30 (1967).

[25] A. C. Eringen, Microcontinuum Field Theory. I. Foundations and Solids (Springer, New York, 1999).

[26] V. I. Erofeev, Wave Processes in Solids with Microstructure (World Scientific, Singapore, 2003).

[27] R. D. Gauthier and W. E. Jahsman, Quest for micropolar elastic-constants, Trans. ASME, J. Appl. Mech. 42(2), 369-374 (1975).

[28] R. D. Gauthier and W. E. Jahsman, Quest for micropolar elastic-constants. 2, Arch. Mech. 33(5), 717-737 (1981).

[29] G. A. Gevorkyan, The basic equations of flexible plates for a medium of Cosserat, Int. Appl. Mech. 3(11), 41-45 (1967).

[30] A.E. Green and P. M. Naghdi, Linear theory of an elastic Cosserat plate, Proc. Cambridge Phil. Soc. Math. Phys. Sci. 63(2), 537-550 (1967).

[31] A. E. Green and P. M. Naghdi, Micropolar and director theories of plates, Q. J. Mech. Appl. Math. 20, 183-199 (1967).

[32] A. E. Green and P. M. Naghdi, The linear elastic Cosserat surface and shell theory, Int. J. Solids Struct. 4(6), 585-592 (1968).

[33] A. E. Green and P. M. Naghdi, On superposed small deformations on a large deformation of an elastic Cosserat surface, J. Elast. 1(1), 1-17 (1971).

[34] A. E. Green and P. M. Naghdi, Derivation of shell theories by direct approach, Trans. ASME, J. Appl. Mech. 41(1), 173-176 (1974).

[35] A.E. Green, P. M. Naghdi, and W. L. Wainwright, A general theory of a Cosserat surface, Arch. Ration. Mech. Anal. 20(4), 287-308 (1965).

[36] E. Grekova and P. Zhilin, Basic equations of Kelvin's medium and analogy with ferromagnets, J. Elast. 64, 29-70 (2001).

[37] E. I. Grigolyuk and I. T. Selezov, Nonclassical Theories of Vibration of Beams, Plates and Shells (in Russ.), Itogi nauki i tekhniki. Mekhanika tverdogo deformiruemogo tela, Vol. 5 (VINITI, Moskva, 1973). 
[38] R. S. Lakes, Experimental microelasticity of two porous solids, Int. J. Solids Struct. 22, 55-63 (1986).

[39] R. S. Lakes, Experimental micro mechanics methods for conventional and negative Poisson's ratio cellular solids as Cosserat continua, Trans. ASME, J. Eng. Mat. Techn. 113, 148-155 (1991).

[40] R. S. Lakes, Experimental methods for study of Cosserat elastic solids and other generalized continua, in: Continuum Models for Materials with Micro-Structure, edited by H. Mühlhaus (Wiley, New York, 1995), pp. 1-22.

[41] A. Libai and J. G. Simmonds, Nonlinear elastic shell theory, Adv. Appl. Mech. 23, 271-371 (1983).

[42] A. Libai and J. G. Simmonds, The Nonlinear Theory of Elastic Shells, 2nd edition (Cambridge University Press, Cambridge, 1998).

[43] R. D. Mindlin, Influence of rotatory inertia and shear on flexural motions of isotropic elastic plates, Trans. ASME, J. Appl. Mech. 18, 31-38 (1951).

[44] R. D. Mindlin and H. F. Tiersten, Effects of couple-stresses in linear elasticity, Arch. Ration. Mech. Anal. 11, 415-448 (1962).

[45] R. Mora and A. Waas, Measurement of the Cosserat constant of circular-cell polycarbonate honeycomb, Philos. Mag. A, Phys. Condens. Matter Struct. Defects Mech. Prop. 80(7), 1699-1713 (2000).

[46] P. Naghdi, The Theory of Plates and Shells, in: Handbuch der Physik, edited by S. Flügge (Springer, Heidelberg, 1972), pp. 425640.

[47] S. A. Nazarov, Asymptotic behavior of the solution of an elliptic boundary value problem in a thin domain, J. Math. Sci. 64(6), 1351-1362 (1993).

[48] P. Neff, A geometrically exact Cosserat shell-model including size effects, avoiding degeneracy in the thin shell limit. Part I: Formal dimensional reduction for elastic plates and existence of minimizers for positive Cosserat couple modulus, Contin. Mech. Thermodyn. 16(6), 577-628 (2004).

[49] P. Neff, The $\Gamma$-limit of a finite strain Cosserat model for asymptotically thin domains and a consequence for the Cosserat couple modulus, Proc. Appl. Math. Mech. 5(1), 629-630 (2005).

[50] P. Neff, The Cosserat couple modulus for continuous solids is zero viz the linearized Cauchy-stress tensor is symmetric, ZAMM 86(11), 892-912 (2006).

[51] P. Neff and K. Chelmiński, A geometrically exact Cosserat shell-model for defective elastic crystals. Justification via $\Gamma$ convergence, Interfaces and Free Boundaries 9, 455-492 (2007).

[52] W. Nowacki, Theory of Asymmetric Elasticity (Pergamon-Press, Oxford et al., 1986).

[53] V. A. Pal'mov, Contribution to Cosserat's theory of plates (in Russ.), Trudy LPI 386, 3-8 (1982).

[54] W. A. Palmow and H. Altenbach, Über eine Cosseratsche Theorie für elastische Platten, Technische Mechanik 3(3), 5-9 (1982).

[55] H. C. Park and R. S. Lakes, Cosserat micromechanics of human bone: strain redistribution by a hydration-sensitive constituent, J. Biomech. 19, 385-397 (1986).

[56] W. Pietraszkiewicz, Consistent second approximation to the the elastic strain energy of a shell, ZAMM 59, 206-208 (1979).

[57] W. Pietraszkiewicz, Finite Rotations and Langrangian Description in the Non-linear Theory of Shells (Polish Sci. Publ, WarszawaPoznań, 1979).

[58] W. Pietraszkiewicz and V. A. Eremeyev, On natural strain measures of the non-linear micropolar continuum, Int. J. Solids Struct. 45, 774-787 (2009).

[59] E. Reissner, On the theory of bending of elastic plates, J. Math. Phys. 23, 184-194 (1944).

[60] E. Reissner, The effect of transverse shear deformation on the bending of elastic plates, Trans. ASME, J. Appl. Mech. 12(11), A69-A77 (1945).

[61] E. Reissner, A note on pure bending and flexure in plane stress including the effect of moment stresses, Arch. Mech. 28(6), 633-642 (1970).

[62] E. Reissner, On sandwich-type plates with cores capable of supporting moment stresses, Acta Mech. 14(1), 43-51 (1972).

[63] E. Reissner, A note on generating generalized two-dimensional plate and shell theories, ZAMP 28, 633-642 (1977).

[64] M. B. Rubin, Cosserat Theories: Shells, Rods and Points (Kluwer, Dordrecht, 2000).

[65] S. O. Sargsyan, On Some Interior and Boundary Effects in thin Plates based on the Asymmetric Theory of Elasticity, in: Theories of Plates and Shells: Critical Review and New Applications, edited by R. Kienzler, H. Altenbach, and I. Ott (Springer, Berlin, 2005), pp. 201-210.

[66] S. O. Sargsyan, Boundary-value problems of the asymmetric theory of elasticity for thin plates, J. Appl. Math. Mech. 72(1), 77-86 (2008).

[67] L. I. Shkutin, Nonlinear models of deformable momental continua (in Russ.), J. Appl. Mech. Tech. Phys. 6, 111-117 (1980).

[68] L. I. Shkutin, Mechanics of Deformations of Flexible Bodies (in Russ.) (Nauka, Novosibirsk, 1985).

[69] J. G. Simmonds, Some Comments on the Status of Shell Theory at the End of the 20th Century: Complaints and Correctives, in: AIAA/ASME/ASCE/AHS/ASC Structures, Structural Dynamics, and Materials Conference and Exhibit, 38th, and AIAA/ASME/AHS Adaptive Structures Forum, Kissimmee, FL, Apr. 7-10, 1997, Collection of Technical Papers, Pt. 3 (A9724112 05-39), (AIAA, 1997), pp. 1912-1921.

[70] S. P. Timoshenko and S. Woinowsky-Krieger, Theory of Plates and Shells (McGraw Hill, New York, 1985).

[71] E. B. Wilson, Vector Analysis, Founded upon the Lectures of J. W. Gibbs (Yale University Press, New Haven, 1901).

[72] P. A. Zhilin, Mechanics of deformable directed surfaces, Int. J. Solids Struct. 12, 635-648 (1976).

[73] P. A. Zhilin, Applied Mechanics. Foundations of the Theory of Shells (in Russ.) (St. Petersburg State Polytechnical University, Saint Petersburg, 2006). 